\title{
A platform for early-career voices
}

In Journal Club articles, early-career researchers share papers that influenced them.

Club format Journal
reminder of the
positive impact
that articles can
have on research
and the course
of individuals'
careers many
years after they
are published

Established researchers have many platforms for their ideas, such as journal articles, department colloquia and conference presentations. By contrast, early-career researchers (ECRs), including graduate students and postdoctoral fellows, often lack a broad audience for their perspectives and insight. This month, Nature Reviews Psychology introduces Journal Club articles, a dedicated format that gives ECRs an opportunity to share their ideas directly with our readership. In each of these short pieces, an ECR discusses a published journal article that has significance for them personally, their research, or the broader field. We hope that these articles will serve as a platform for the ideas of researchers who are shaping the future of the field.

Although typical journal club groups in a laboratory or department might focus on brand-new scientific findings, our format is more flexible. In this way, our Journal Club format is a reminder of the positive impact that articles can have on research and the course of individuals' careers many years after they are published. Perhaps a review paper assigned as a reading in an undergraduate psychology class inspires a student to change their specialization and pursue a graduate degree in psychology. Discussing a paper that introduces a new methodology in a graduate seminar might inspire an ECR to apply that method in their own area, forging a new interdisciplinary research direction. A heated laboratory meeting debate about the implications of an empirical finding could prompt a direct or conceptual replication of the original study. Many of these impacts can easily be invisible to the authors of the original paper. Journal Club articles bring them to the forefront through the first-person perspective of an ECR.

Each Journal Club article is drafted by the ECR and then revised on the basis of feedback from one of the journal editors. Through this process we aim to preserve each ECR's unique voice while providing the kind of editorial feedback and writing guidance that we provide to all of our authors. ECRs are asked to include not only the impact of their chosen paper on the field, but also any significance that paper has for their own personal or professional outlook.

To kick off this format, our February issue includes four Journal Club articles across a range of topics in psychology. We hope that ECRs and established researchers alike find something to spark their interest and learn something new from these pieces.

Sibele Aquino highlights a 2015 review paper on the difference between pleasure gained from purchasing experiences and purchasing goods. Aquino explores how the paper's conclusions have held up in the intervening years and how subsequent research has questioned a strict dichotomy between these two types of purchases.

Rebecca Canale writes about a foundational 1996 paper that established that young infants can learn statistical regularities presented in spoken syllables. Canale describes how this paper opened the door to research on statistical learning in children on the autism spectrum, pointing to open questions regarding the impact of variability in language and cognitive abilities.

Sakshi Ghai examines a 2020 paper that quantified scientific innovation in doctoral dissertations relative to the race, gender, and subsequent career progression of each author. Ghai highlights that novel research topics that push disciplinary boundaries are harder to integrate with existing work, yet are key to increasing diversity and progress in the field.

Jonathan Tsay describes findings from a 2014 paper that showed distinct explicit and implicit contributions to motor adaptation with impaired visual feedback. Tsay first read this paper while working as a licensed physical therapist and it inspired him to begin graduate studies in the laboratory of one of the paper's authors.

Together, these Journal Club articles showcase a variety of influences on ECRs in a range of fields. We hope these pieces inspire all psychologists to think about the life of their papers after they are published: a paper written this year might provide inspiration for a future psychologist to make their mark on the field next month or a few decades from now.

If you are a graduate student or postdoctoral fellow who would like to highlight a paper in a future Journal Club article, let us know! Send us an email (nrpsych@ nature.com) with your name, career stage, affiliation, the paper you would like to write about and a brief paragraph about how the paper has influenced you or your field. 